\title{
2,3-二氢喹唑啉-4(1H)-酮类化合物的合成研究进展
}

\author{
张晓鹏* 朱妍洁朱奕崧李政伟张贵生 \\ (河南师范大学化学化工学院 绿色化学介质与反应教育部重点实验室 河南省有机功能分子 \\ 与药物创新重点实验室 精细化学品绿色制造河南省协同创新中心 新乡 453007)
}

\begin{abstract}
摘要 2,3 -二氢喹唑啉-4(1H)-酮是一类重要的含氮稠杂环化合物, 具有广泛的药理及生物活性, 在药物合成与研发领 域有着重要应用价值, 因而其合成方法也倍受人们关注. 归纳总结了 2,3-二氢喹唑啉-4(1H)-酮类化合物的合成研究进 展, 主要介绍了以邻氨基苯甲酰胺、靛红酸䣶、邻硝基苯甲酰胺、邻叠氮基苯甲酰胺、邻溴苯甲酰胺、邻溴苯甲腈、 邻氨基苯甲酸、邻氨基苯甲腈、邻氨基 $N$-甲基 $-N$-丙二烯基苯甲酰胺、 $N$-烷基苯胺等为起始原料的 2,3-二氢喹唑啉-4(1H)酮类化合物的合成研究进展概况及其反应机理. 最后对该类化合物的合成研究进展进行了总结, 并对其发展前景进行 了展望.
\end{abstract}

关键词 2,3-二氢喹唑啉-4(1H)-酮; 邻氨基苯甲酰胺; 靛红酸酐; $N$-烷基苯胺; $\mathrm{C}-\mathrm{H}$ 活化功能化; 钯催化

\section{Advances in Synthesis of 2,3-Dihydroquinazolin-4(1H)-ones}

\author{
Zhang, Xiaopeng* Zhu, Yanjie Zhu, Yisong Li, Zhengwei Zhang, Guisheng
}

(Collaborative Innovation Center of Henan Province for Green Manufacturing of Fine Chemicals, Henan Key Laboratory of Organic Functional Molecule and Drug Innovation, Key Laboratory of Green Chemical Media and Reactions, Ministry of Education, School of Chemistry and Chemical Engineering, Henan Normal University, Xinxiang 453007)

\begin{abstract}
Dihydroquinazolin-4(1H)-one compounds are an important class of nitrogen-containing fused heterocycles, which possess a wide range of pharmacological and biological activities and have important applications in the fields of synthesis and research \& development of drugs. Therefore, its synthetic methods have also attracted considerable attention. In this paper, the main advances in the synthesis of 2,3-dihydroquinazolin-4(1H)-ones and their proposed reaction mechanisms from the raw materials such as $o$-aminobenzamides, isatoic anhydrides, $o$-nitrobenzamides, $o$-azidobenzamide, $o$-bromobenzamide, $o$-bromobenzonitrile, $o$-aminobenzoic acids, $o$-aminobenzonitrile, $o$-amino- $N$-(propa-1,2-dienyl)benzamides, and $\mathrm{N}$-alkyl anilines were introduced and reviewed, respectively. Finally, the synthesis of these compounds was summarized and the prospect of their development was prospected.

Keywords 2,3-dihydroquinazolin-4(1H)-one; $o$-aminobenzamides; isatoic anhydrides; $N$-alkyl anilines; $\mathrm{C}-\mathrm{H}$ activation \& functionalization; palladium catalysis
\end{abstract}

2,3-二氢喹唑啉-4(1H)-酮是一类重要的含氮稠杂环 化合物，具有抗癌 ${ }^{[1 \sim 3]}$ 、抗肿瘤 ${ }^{[4 \sim 6]}$ 、治疗高血压 ${ }^{[7 \sim 9]}$ 、

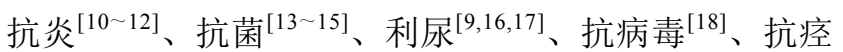
挛 $^{[19] 、 \text { 抑制㾏原虫增殖 }}{ }^{[20]}$ 、驱虫 ${ }^{[21]}$ 、镇痛 ${ }^{[12]}$ 等作用以 及抑制组织蛋白酶 $B \& \mathrm{H}^{[22]}$ 、胆碱酯酶 ${ }^{[23,24]}$ 、凝血因子 $\mathrm{Xa}^{[25]}$ 、肌苷 $5^{\prime}-$ 一磷酸脱氢酶 ${ }^{[26]}$ 、蛋白激酶 $\mathrm{C}-\theta^{[27]}$ 、瞬 时受体电位通道 $\mathrm{M} 2^{[28]}$ 等广泛的药理及生物活性; 此外, 该类化合物也很容易被氧化成其喹唑啉-4(3H)-酮类似 物, 后者通常作为重要模块用于构建天然产物 ${ }^{[29 \sim 31]}$ 及
药物分子 ${ }^{[32 ~ 34]}$.

由于 2,3-二氢喹啉-4(1H)-酮类化合物具有广泛且重 要的生物活性及应用价值, 其合成方法也自然引起了人 们的极大兴趣和关注. 根据国内外的研究动态, 对其主 要合成方法进行了总结.

\section{1 以邻氨基苯甲酰胺为底物}

\section{1 邻氨基苯甲酰胺与醛/酮反应}

在催化剂作用下邻氨基苯甲酰胺与醛/酮直接环合

* Corresponding author. E-mail: zhangxiaopengv@sina.com

Received March 14, 2019; revised April 11, 2019; published online April 26, 2019.

Project supported by the National Natural Science Foundation of China (Nos. 21772033, U1604285) and the Program of Introducing Talents of Discipline to Universities (111 Project, No. D17007).

国家自然科学基金(Nos. 21772033, U1604285)、高等学校学科创新引智计划(111 计划, No. D17007)资助项目. 
是最常见、最简便的一种合成 2,3-二氢喹啉-4(1H)-酮类 化合物的方法(Eq. 1) ${ }^{[35 \sim 37]}$.<smiles>[R1]Nc1ccccc1C(=O)NN[Z2](C)=O</smiles>

该方法反应体系简单、反应条件温和、用时较短、 操作方便、产率良好. 所用催化剂主要是质子酸类催化 剂(如 $\mathrm{TsOH} 、 \mathrm{H}_{2} \mathrm{SO}_{4}$ 、浓 $\mathrm{HNO}_{3} / \mathrm{HCl} 、 \mathrm{HCOOH}$ 等)和路 易斯酸类催化剂[如 $\mathrm{Sc}(\mathrm{OTf})_{3} 、 \mathrm{Yb}(\mathrm{OTf})_{3} 、 \mathrm{ZrCl}_{2} 、 \mathrm{HgCl}_{2}$ 、 $\mathrm{H}_{3} \mathrm{BO}_{3}$ 等 $]^{[35]}$, 此外, 碱 [如 $\mathrm{NaOH} 、 \mathrm{NaOEt}$ 及铵盐如 $\mathrm{NH}_{4} \mathrm{Cl} 、 \mathrm{TBAHS}$ (四丁基硫酸氢铵)、 $\mathrm{Ce}\left(\mathrm{NH}_{4}\right)_{2}\left(\mathrm{NO}_{3}\right)_{6}$ 等] 也可以用作催化剂 ${ }^{[35]}$.

若在手性条件下进行该反应, 可方便地实现 2,3-二 氢喹啉-4(1H)-酮类化合物的对映选择性合成(Scheme 1). 例如在手性催化剂 $(S)$ - 葱修饰的联萗酚磷酸酯 $[(S)$ anthracenyl-modified TRIP, (S)-AMTRIP]存在下, (异)丙 醛、异丁醛等脂肪醛, 苯甲醛和苯乙醛等芳香醛以及 2,3-二酮二氢吲哚等均能与邻氨基苯甲酰胺顺利发生环 合反应，可以 $67 \% \sim 96 \%$ 的产率得到 $R$ 构型的目标产物， 其绝大部分 $e e$ 值在 $89 \% \sim 98 \%$ 之间 ${ }^{[36]}$; 而当 $\mathrm{Sc}(\mathrm{OTf})_{3} /$ 手性双噁唑啉含氟配体[chiral fluorous bis(oxazolines),
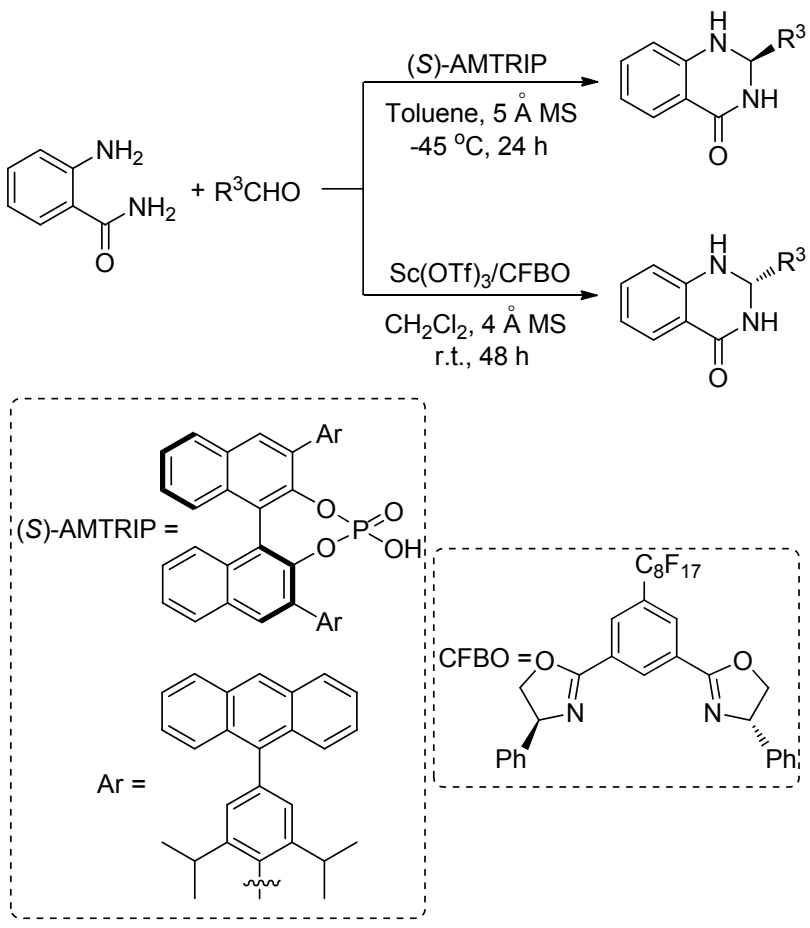

图式 1 手性条件下邻氨基苯甲酰胺与醛/酮反应

Scheme 1 Reaction of $o$-aminobenzamides with aldehydes/ ketones under chiral conditions

CFBO]组成的催化体系用于该反应时, 一系列苯系芳 醛、2-吡啶甲醛、1-荎甲醛等芳香醛及环己基甲醛均可
顺利与邻氨基苯甲酰胺发生反应，能以 $76 \%$ ～94\%的收 率得到 $S$ 构型的目标产物, ee 值在 $88 \% \sim 98 \%$ 之间 ${ }^{[37]}$.

尽管所用的催化剂种类繁多，但其环合机理大致相 同(Scheme 2). 首先, 醛/酮羰基在催化剂活化作用下接 受邻氨基苯甲酰胺苯环上氨基氮的亲核进攻生成羟基 加成物 I; 紧接着, I 在催化剂作用下脱水生成席夫碱 II; 然后，II 经酰胺基中氮对亚胺碳亲核进攻的分子内环合 作用生成目标产物 III $^{[35,38,39]}$.

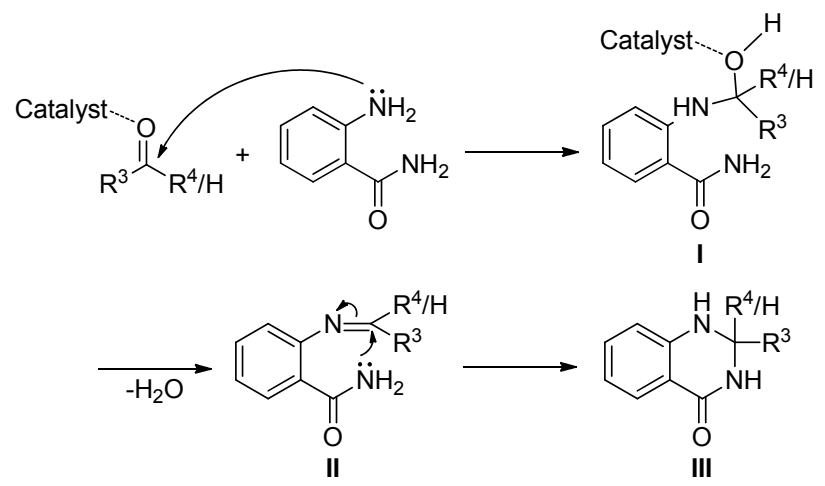

图式 2 邻氨基苯甲酰胺与醛/酮反应的机理

Scheme 2 Proposed mechanism of the cyclocondensation of $o$-aminobenzamides with aldehydes/ketones to 2,3-dihydroquinazolin-4-(1H)-ones

\section{2 邻氨基苯甲酰胺与仲胺反应}

在过量的过硫酸氢钾复合盐(Oxone)作用下邻氨基 苯甲酰胺与仲胺环合也可用来制备 2,3-二氢喹啉-4(1H)酮类化合物(Eq. 2) ${ }^{[40]}$.

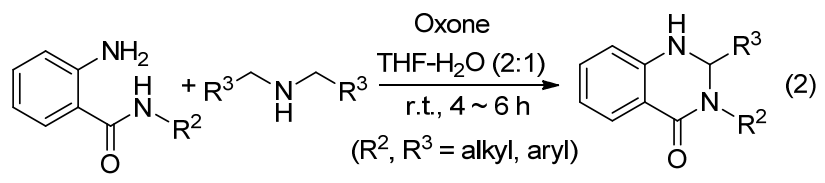

该反应在室温条件下就能顺利进行，目标产物收率 在 65\% 92\%之间. Eq. 2 中的 $\mathrm{R}^{2} 、 \mathrm{R}^{3}$ 既可以是脂肪基， 也可以是芳香基. 但该方法所用原料之一的胺仅限于 仲胺, 且原子经济性较差.

以 $\mathrm{N}$-苯基邻氨基苯甲酰胺与二芐胺的环合为例, 其反应机理可描述如 Scheme 3 所示. 首先, 二芐胺在过 硫酸氢钾复合盐作用下生成相应的席夫碱氮氧化物 $\mathbf{I}$, 然后 I 接受 $N$-苯基邻氨基苯甲酰胺苯环上氨基氮对其对 亚胺碳的亲核进攻生成加成产物 II，随后 II 脱去 $N$-芐基 差弪胺生成相应的席夫碱 III, 最后 III 经酰胺基中氮对亚 胺碳亲核进攻的分子内环合作用生成目标产物 IV.

\section{3 邻氨基苯甲酰胺与乙烯基卤代物反应}

在 $\mathrm{CuI}$ 催化下, 邻氨基苯甲酰胺可与乙烯基卤代物 反应生成 2,2-二取代的 2,3-二氢喹啉-4(1H)-酮类化合物. 


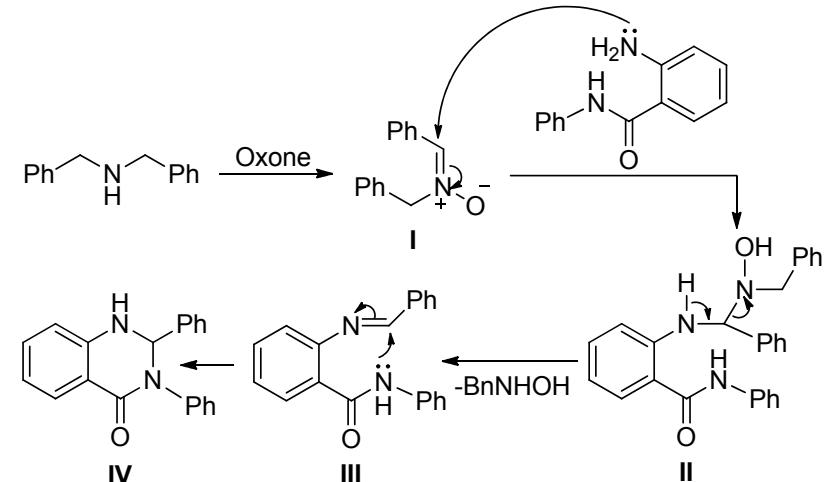

图式 3 邻氨基苯甲酰胺与仲胺环合生成 2,3-二氢喹唑啉4(1H)-酩的机理

Scheme 3 Proposed mechanism of the cyclocondensation of $o$-aminobenzamides with $s e c$-amines to 2,3-dihydroquinazolin4-(1H)-ones

其中的乙烯基碘代物也可由炔烃经碘氢化反应(Hydroiodination)制得, 无需分离即可与邻氨基苯甲酰胺类化 合物进行 “一锅法” 反应制得目标产物(Scheme 4) ${ }^{[41]}$.

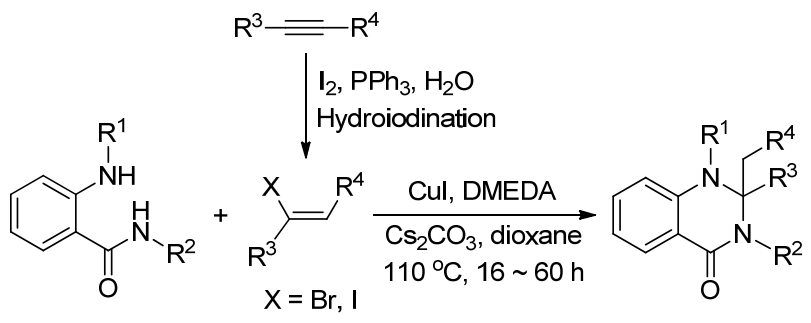

图式 4 邻氨基苯甲酰胺与乙烯基卤代物反应

Scheme 4 Reaction of $o$-aminobenzamides with vinyl halides

利用该方法可制备 2,2-二取代的 2,3-二氢喹啉$4(1 H)$-酮，分离收率在 $45 \% \sim 93 \%$ 之间. 但该反应需要 用 $N, N^{\prime}$-二甲基-1,2-乙二胺(DMEDA)作配体，而且反应 时间很长.

该反应的机理如 Scheme 5 所示. 首先邻氨基苯甲 酰胺中的酰胺基与乙烯基卤代物反应生成烯胺中间体 $\mathbf{I}$, I 经分子内重排可与 II 互变, 然后 I/II 再经分子内的氢 胺化反应环合生成 2,2-二取代的 2,3-二氢喹啉-4(1H)-酮 (III) ${ }^{[41]}$.

\section{4 邻氨基苯甲酰胺与炔/炔醇反应}

邻氨基苯甲酰胺在催化剂作用下可与末端炔烃或 炔醇类化合物发生双氢胺化反应环合生成 2,2-二取代的

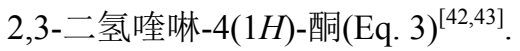

当末端炔烃参与反应时, 所用的催化剂为 $\mathrm{Ph}_{3} \mathrm{PAu}-$ $\mathrm{NTf}_{2}$, 目标产物收率良好, 在 $75 \% \sim 98 \%$ 之间; 当炔醇 参与该反应时, 所用的催化剂为 $\mathrm{PtBr}_{2}$ 或 $\mathrm{Au}\left(\mathrm{PPh}_{3}\right) \mathrm{Cl} /$ AgOTf，目标产物收率在 52\% 97\%之间. 利用该方法 可直接高产率合成 2,2-二取代的 2,3-二氢喹啉-4(1H)-酮,
但反应时间较长，所用催化剂昂贵.

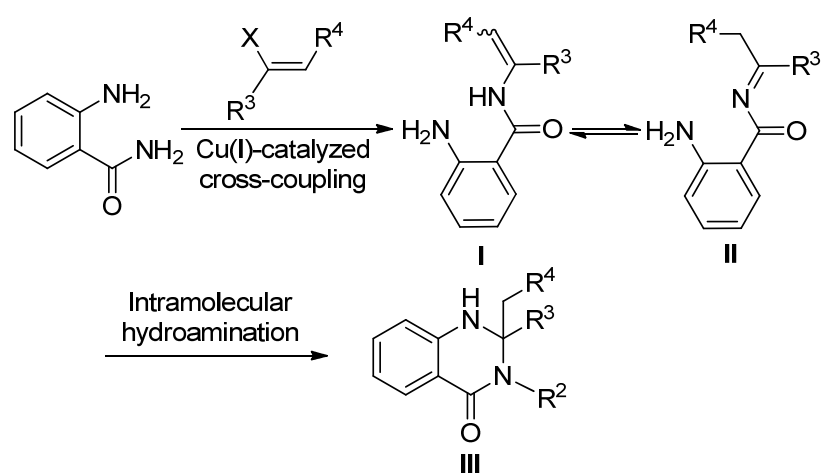

图式 5 邻氨基苯甲酰胺与乙烯基卤代物环合生成 2,3-二氢喹 唑啉-4(1H)-酮的机理

Scheme 5 Proposed mechanism of the cyclocondensation of $o$-aminobenzamides with vinyl halides to 2,3-dihydroquinazolin-4-(1H)-ones

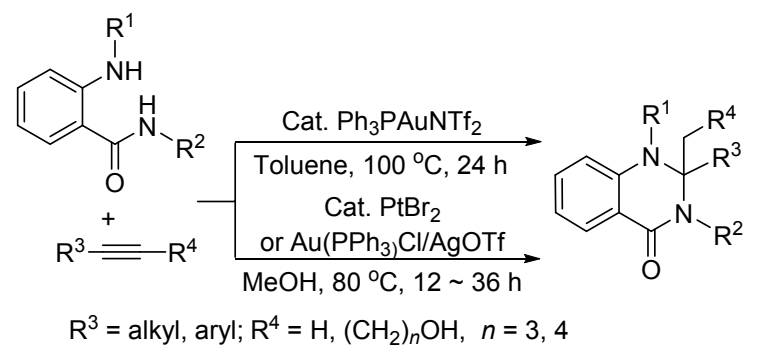

其中端炔参与反应的机理如 Scheme 6 所示. 首先炔 烃在催化剂作用下生成络合物 $\mathbf{I}$, 然后与邻氨基苯甲酰 胺苯环上的胺基发生马尔科夫尼科夫式的氢胺化反应 生成烯胺中间体 II, 随后 II 再与酰胺中胺基发生马氏氢 胺化反应环合生成目标产物 III $^{[42,43]}$.

炔醇参与反应的机理如 Scheme 7 所示. 首先炔醇中 炔基与催化剂作用生成络合物 $\mathbf{I}$, 随后羟基通过对炔基 的马氏氢烷氧基化作用环合生成 II，进而生成 III，然后 邻氨基苯甲酰胺中苯环上的氨基在催化剂作用下与 III 发生马氏氢胺化加成反应生成 IV, 进而再转变成 $\mathbf{V}$, 随 后 $\mathbf{V}$ 再进一步异构化为席夫碱类中间体 VI，最后 VI 经 分子内酰胺基对席夫碱的加成环合反应生成目标产物 VII ${ }^{[43]}$.

\section{2 以靛红酸酐为底物}

\section{1 靛红酸酐与胺和醛/酮反应}

靛红酸酐与胺、醛/酮反应是文献中报道的合成 2,3二氢喹啉-4(1H)-酮类化合物的另一个主要方法，通常有 两条实现途径: 其一，靛红酸酐首先与胺脱羧缩合生成 邻氨基苯甲酰胺, 然后邻氨基苯甲酰胺在催化剂如 $\mathrm{TsOH} 、 \mathrm{AcOH}$ 等作用下与醛/酮环合制得目标产物 (Scheme 8$)^{[44,45]}$. 该途径需经两步反应才能得到目标产 


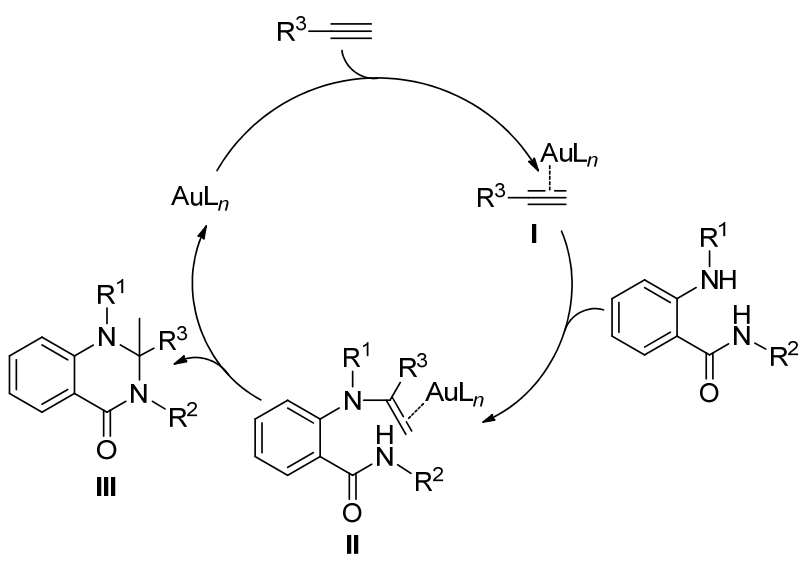

图式 6 邻氨基苯甲酰胺与端炔环合生成 2,3-二氢喹唑啉4(1H)-酮的机理

Scheme 6 Proposed mechanism of the cyclocondensation of $o$-aminobenzamides with terminal alkynes to 2,3-dihydroquinazolin-4-(1H)-ones

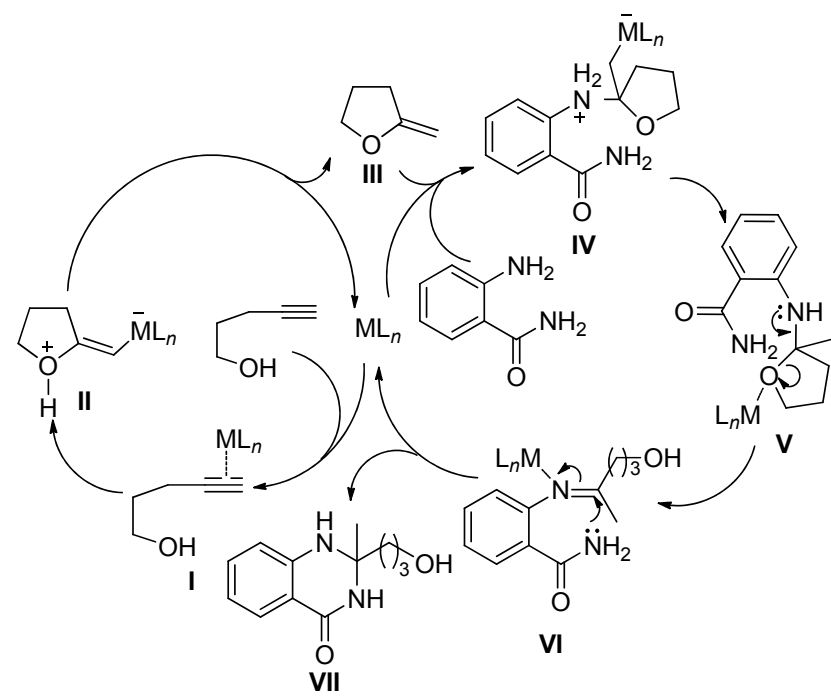

图式 7 邻氨基苯甲酰胺与炔醇环合生成 2,3-二氢喹唑啉4(1H)-酤的机理

Scheme 7 Proposed mechanism of the cyclocondensation of $o$-aminobenzamides with alkynols to 2,3-dihydroquinazolin-4(1H)-ones

物, 操作相对繁琐, 且目标产物收率较低; 其二, 将上 述两步反应串联起来, 即靛红酸酐、胺和醛/酮经 “一锅 法” 的三组分串联反应直接得到目标产物 (Eq. $4)^{[35,45 \sim 48]}$. 该方法是由靛红酸䣶合成 2,3-二氢喹啉4(1H)-酮类化合物的主要途径, 步骤简短, 操作方便, 用时较短, 绝大部分目标产物收率良好, 常用的催化剂 有 $\mathrm{TsOH} 、 \mathrm{AcOH} 、 乙 二$ 二胺二乙酸(EDDA)、 $\mathrm{Cu}(\mathrm{OTf})_{2}$ 、 $\mathrm{Yb}(\mathrm{OTf})_{3} 、 \mathrm{Ga}(\mathrm{OTf})_{3} 、 \mathrm{Sc}(\mathrm{OTf})_{3} 、 \mathrm{SrCl}_{2} \bullet 6 \mathrm{H}_{2} \mathrm{O} 、 \mathrm{KAl}\left(\mathrm{SO}_{4}\right)_{2} \bullet$ $12 \mathrm{H}_{2} \mathrm{O} 、 \mathrm{I}_{2} 、$ Citric acid、 $\mathrm{SSA}$ (磺化硅胶)等以及 $\mathrm{Fe}_{3} \mathrm{O}_{4}$ 、 $\mathrm{Al} / \mathrm{Al}_{2} \mathrm{O}_{3} 、 \mathrm{CuO} 、 \mathrm{In}_{2} \mathrm{O}_{3}$ 、羟基磷灰石(HAP)等纳米粒 子[35,45 48].
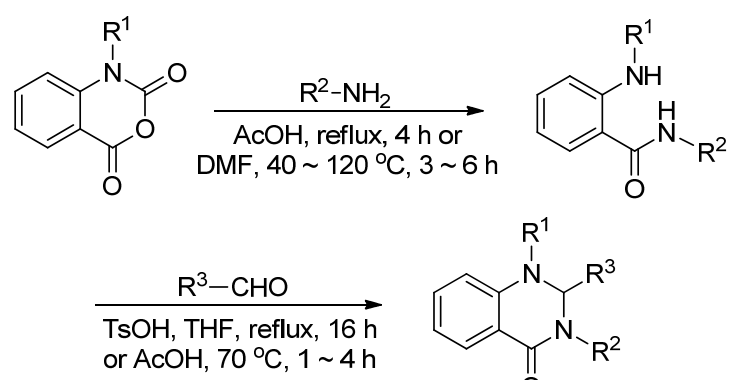<smiles>[R]C1N([R])C(=O)c2ccccc2N1[R]</smiles>

图式 8 靛红酸酐与胺、醛/酮分步反应

Scheme 8 Stepwise reaction of isatoic anhydrides with amines and aldehydes/ketones

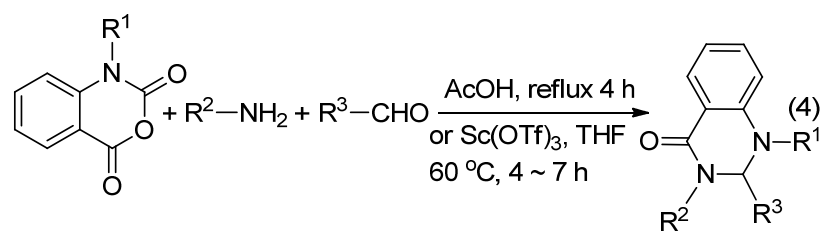

“一锅法” 的三组分串联反应生成 2,3-二氢喹啉$4(1 H)$-酮的机理如 Scheme 9 所示 ${ }^{[35,46,47]}$. 首先, 靛红酸 酐中的羰基在催化剂作用下接受胺的亲核进攻脱去 $\mathrm{CO}_{2}$ 生成邻氨基苯甲酰胺 $\mathbf{I}$, 然后 $\mathbf{I}$ 中的氨基再对醛/酮 中被催化剂活化了的羰基进行亲核进攻，生成差基加成 物 II, 随后 II 脱水生成席夫碱 III, 最后 III 经分子内环 合作用生成目标产物 IV.
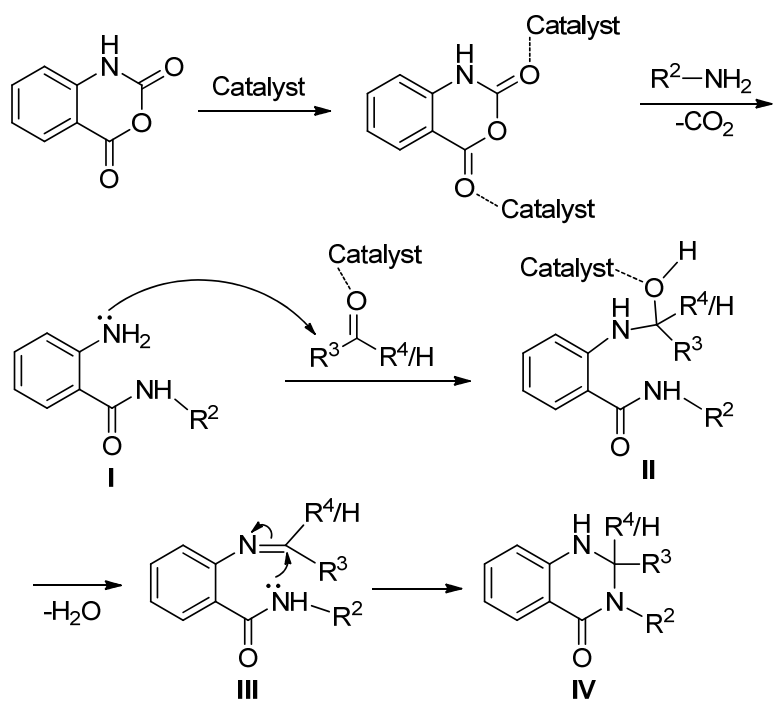

图式 9 靛红酸䣶与胺、醛 “一锅法” 反应生成 2,3-二氢喹唑 啉-4(1H)-酮的机理

Scheme 9 Proposed mechanism of one-pot reaction of isatoic anhydride with amines and aldehydes/ketones to 2,3-dihydroquinazolin-4-(1H)-ones

\section{2 靛红酸酐与席夫碱反应}

靛红酸酐在微波辐射下或无溶剂条件下与席夫碱 
反应生成 2,3-二芳基取代的 2,3-二氢喹啉-4(1H)-酮类化 合物. 当用微波辐射加热时，反应可在 $2 \sim 4 \mathrm{~min}$ 内完成， 目标产物产率在 $60 \% \sim 90 \%$ 之间 ${ }^{[49,50]}$; 无溶剂条件下, 该反应各原料在 $\mathrm{NaOH}$ 存在下在研钵中研磨均匀后再 转移入反应瓶中加热反应 $30 \mathrm{~min}$, 即可以 $80 \%$ 96\%的 收率得到目标产物(Eq. 5) ${ }^{[51]}$.

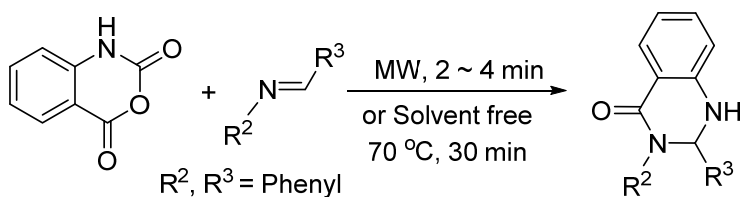

相应地, 若靛红酸酐与环状席夫碱如 3,4-二氢异喹 啉反应, 则可方便地得到四元稠杂环的 2,3-二氢喹啉$4(1 H)$-酮类化合物 ${ }^{[52,53]}$, 从而为吴茱英次碱等多元稠杂 环类 2,3-二氢喹啉-4(1H)-酮类化合物的合成开辟出一条 新途径. 其反应过程为 3,4-二氢异喹啉中亚胺氮对靛红 酸酐中羰基亲核加成导致其开环生成中间体 $\mathbf{I}$, 然后 $\mathbf{I}$ 中氮再对其亚胺碳亲核进攻环合同时脱去 $\mathrm{CO}_{2}$ 即可得 到目标产物，反应式如 Scheme 10 所示.

\section{3 靛红酸酎与胺和苄醇反应}

在 $\mathrm{HBr} / \mathrm{DMSO}^{[54]}$ 或 $\mathrm{I}_{2} / \mathrm{K}_{2} \mathrm{CO}_{3}{ }^{[55]}$ 促进下, 靛红酸酐与 胺和苠醇反应能在短时间内高收率(90\% 96\%)地得到 2-苯基取代的 2,3-二氢喹啉-4(1H)-酮类目标产物(Eq. $6)$.

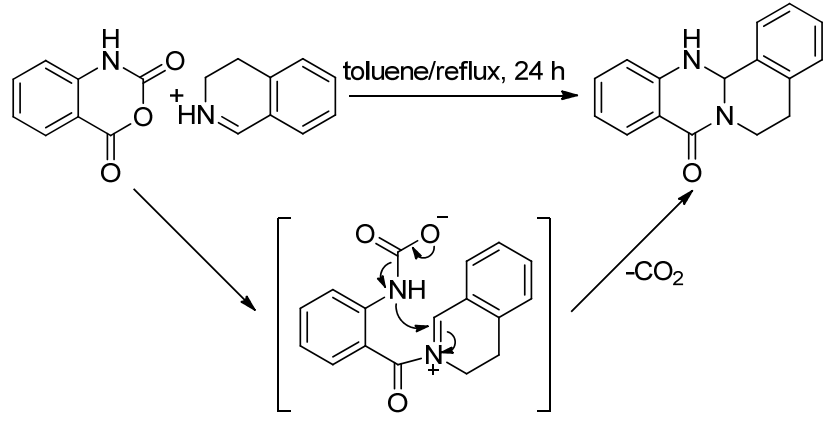

图式 10 靛红酸酐与 3,4-二氢异喹啉反应

Scheme 10 Reaction of isatoic anhydride with 3,4-dihydroisoquinoline

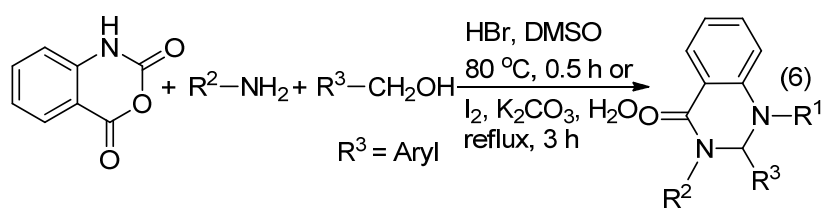

上述两反应体系尽管所用原料相同，但其反应机理 却不尽相同，具体情况如 Scheme 11 所示. 首先，靛红酸 酐在胺作用下脱去 $\mathrm{CO}_{2}$ 生成邻氨基苯甲酰胺(I). 当在 $\mathrm{HBr} / \mathrm{DMSO}$ 体系中时, 该反应以 Path A 的路径进行, 即 DMSO 与 $\mathrm{HBr}$ 反应先后经 IA、IIA 生成溴化二甲基溴 化硫(IIIA), 然后 IIIA 与苄醇反应生成烷氧基锍离子 (IVA)，接着 IVA 与 I 反应，在脱去 DMSO 和 $\mathrm{HBr}$ 的同

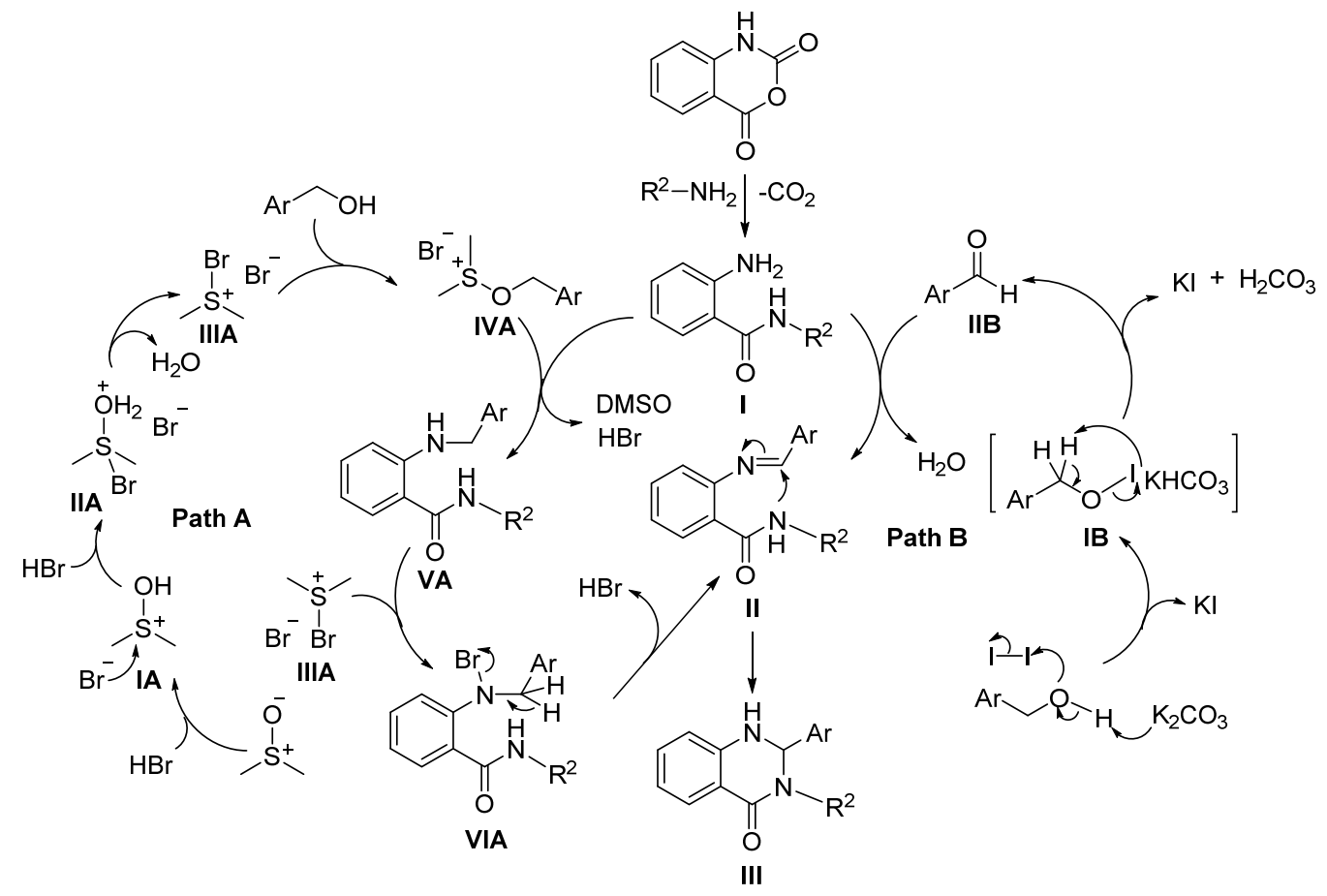

图式 11 靛红酸酐与胺、茮醇反应生成 2,3-二氢喹唑啉-4(1H)-酮的机理

Scheme 11 Proposed mechanism of reaction of isatoic anhydride with amines and benzyl alcohols to 2,3-dihydro-quinazolin4-(1H)-ones 
时生成 $N$-芐基邻氨基苯甲酰胺中间体 VA, 随后 VA 在 IIIA 作用下溴化生成 VIA, 紧接着其再经 E2 消除反应 脱去 $\mathrm{HBr}$ 生成相应的席夫碱类中间体 II, 最后 II 再经分 子内的环合反应生成目标产物 $\mathbf{I I I}{ }^{[54]}$; 当在 $\mathrm{I}_{2} / \mathrm{K}_{2} \mathrm{CO}_{3}$ 体 系中时, 反应则以 Path B 的方式来进行: 在 $\mathrm{K}_{2} \mathrm{CO}_{3}$ 存在 下芐醇与 $\mathrm{I}_{2}$ 反应生成中间体 $\mathbf{I B}$ 和 $\mathrm{KHCO}_{3}$, 随后经消除 反应生成芳醛 IIB, 然后 IIB 再与 I 反应生成 II, 然后 II 再经分子内的环合反应生成目标产物 $\mathbf{~ I I I ~}^{[55]}$.

\section{3 以邻硝基苯甲酰胺为底物}

在还原体系中, 邻硝基苯甲酰胺可与醛/酮反应直 接生成 2,3-二氢喹啉-4(1H)-酮类化合物, 收率在 $60 \%$ $94 \%$ 之间(Eq. 7). 所用还原体系有 $\mathrm{Fe} / \mathrm{AcOH}^{[56]} 、 \mathrm{TiCl}_{4} /$ $\mathrm{Zn}^{[57]} 、 \mathrm{SmI}_{2}{ }^{[58]} 、 \mathrm{Sm} / \mathrm{I}_{2}$ (Cat. $)^{[59]}$ 等.

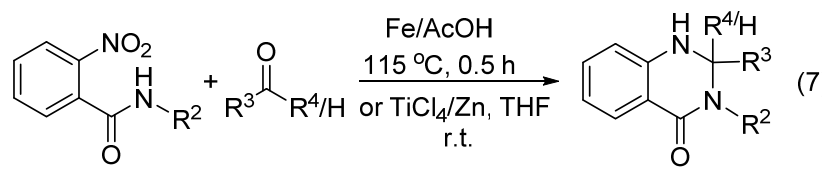

当所用的还原体系为 $\mathrm{Fe} / \mathrm{CH}_{3} \mathrm{COOH}^{[56]} 、 \mathrm{TiCl}_{4} / \mathrm{Zn}^{[57]}$ 、 $\mathrm{SmI}_{2}{ }^{[58]}$ 等时, 其反应历程为: 邻硝基苯甲酰胺在还原剂 存在下首先被还原为邻氨基苯甲酰胺, 然后再与醛/酮 环合生成目标产物; 当还原体系为 $\mathrm{Sm} / \mathrm{I}_{2}$ (Cat.)时, 作者 提出的反应机理与上述不同, 具体历程如 Scheme 12 所 ${ }^{\text {示 }}{ }^{[59]}$. 金属缷、三价钐等与 $\mathrm{I}_{2}$ 作用生成低价钐, 然后其 将邻硝基苯甲酰胺经邻亚硝基苯甲酰胺(I)还原为中间 体 II, 随后 II 再与醛/酮反应加成产物 III, 紧接着 III
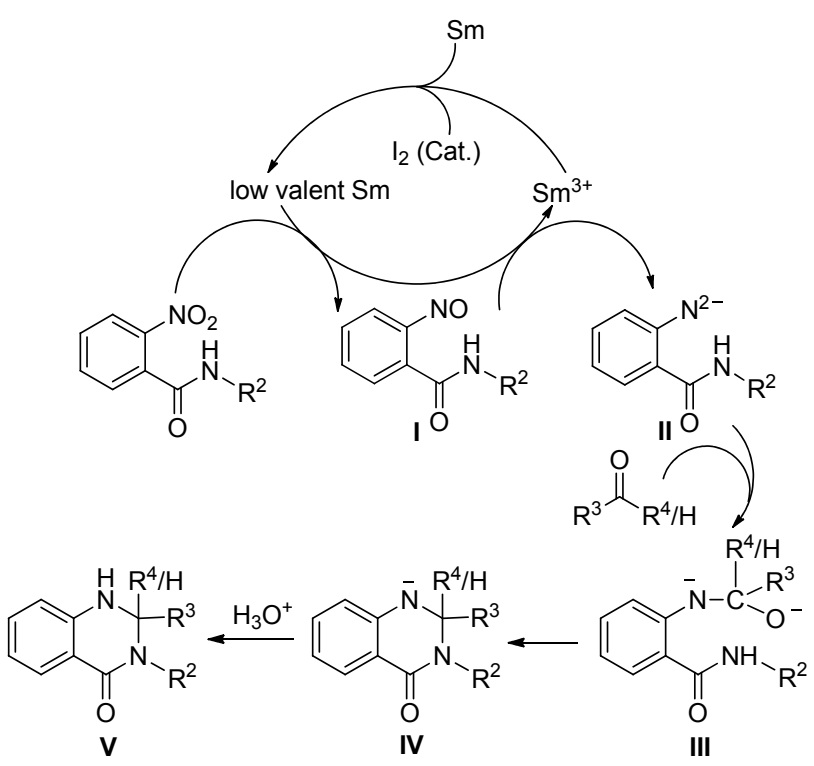

图式 $12 \mathrm{Sm} / \mathrm{I}_{2}$ (Cat.)作用下邻硝基苯甲酰胺与醛/酮反应生成 2,3-二氢喹唑啉-4(1H)-酮的机理

Scheme 12 Proposed mechanism of reaction of $o$-nitrobenzamides with aldehydes/ketones to 2,3-dihydroquinazolin-4(1H)-ones promoted by $\mathrm{Sm} / \mathrm{I}_{2}$ (Cat.)

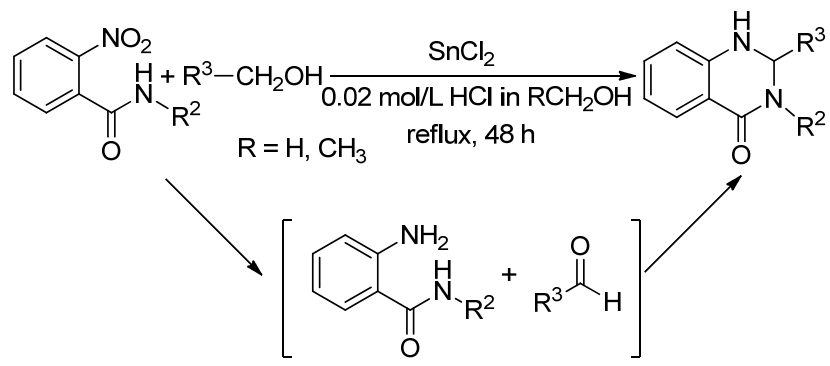

图式 13 邻硝基苯甲酰胺与甲醇/乙醇反应

Scheme 13 Reaction of $o$-nitrobenzamides with methanol/ethanol

再经环合反应生成 IV, 然后在酸存在下 IV 进一步转变 成目标产物 $\mathbf{V}$.

在过量 $\mathrm{SnCl}_{2}$ (10 equiv.)存在下, 邻硝基苯甲酰胺也 可与甲醇/乙醇的稀盐酸溶液反应制得相应的 2,3-二氢 喹啉-4(1H)-酮类化合物 ${ }^{[60]}$. 该方法以甲醇/乙醇替代相 应的羰基化合物，所用起始原料更加简单，但目标产物 收率普遍不高，在 $50 \% \sim 73 \%$ 之间，而且反应时间较长， 需要 48 h. 该反应历程如 Scheme 13 所示. 在 $\mathrm{SnCl}_{2}$ 存在 下邻硝基苯甲酰胺与醇首先转化成相应的邻氨基苯甲 酰胺与醛, 然后两者再环合生成目标产物.

\section{4 以邻叠氮基苯甲酰胺为底物}

在 $\mathrm{SmI}_{2}{ }^{[61]} 、 \mathrm{Sm} / \mathrm{I}_{2}$ (Cat.) ${ }^{[59]}$ 等作用下, 邻叠氮基苯甲 酰胺可与醛或 2-戊酮反应，以 $69 \% \sim 89 \%$ 的收率得到目 标产物; 而当芳香酮用于该反应时, 即使在甲醇中回流 $20 \mathrm{~h}$, 也未能得到相应的目标产物. 该反应需要在无水、 氮气保护条件下进行(Eq. 8).

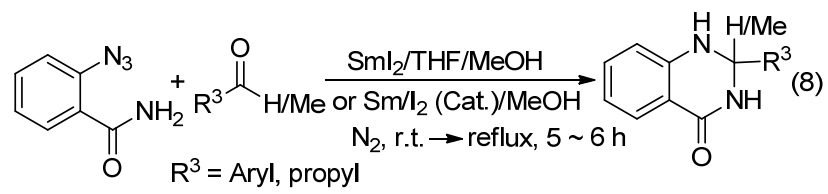

以在 $\mathrm{SmI}_{2}$ 作用下为例, 其反应机理如 Scheme 14 所 示. 邻叠氮基苯甲酰胺首先与 $\mathrm{SmI}_{2}$ 作用生成互变异构中 间体 I 和 II, 然后再与醛/酮反应生成目标产物 III ${ }^{[61]}$.

\section{5 以邻溴苯甲酰胺为底物}

在 $\mathrm{CuI}^{[62]} 、 \mathrm{CuCl}_{2}{ }^{[63]}$ 催化下, 邻溴苯甲酰胺可与芳香 醛在合适氮源如 $\mathrm{TMSN}_{3}$ (叠氮三甲基硅烷)、 $\mathrm{NH}_{3} \cdot \mathrm{H}_{2} \mathrm{O}$ 等 存在下发生反应, 以 $68 \% \sim 78 \%$ 的收率得到 2-芳基取代 的 2,3-二氢喹啉-4(1H)-酮类化合物(Eq. 9).

以 $\mathrm{TMSN}_{3}$ 作氮源为例, 该反应的机理如 Scheme 15 所示 ${ }^{[62]}$. 首先, $\mathrm{CuI}$ 与邻溴苯甲酰胺发生氧化加成反应生 成配合物 I, 然后与 $\mathrm{TMSN}_{3}$ 作用生成配合物 II, II 在含 少量水的 DMSO 作用下经铜催化的脱氮反应生成配合 


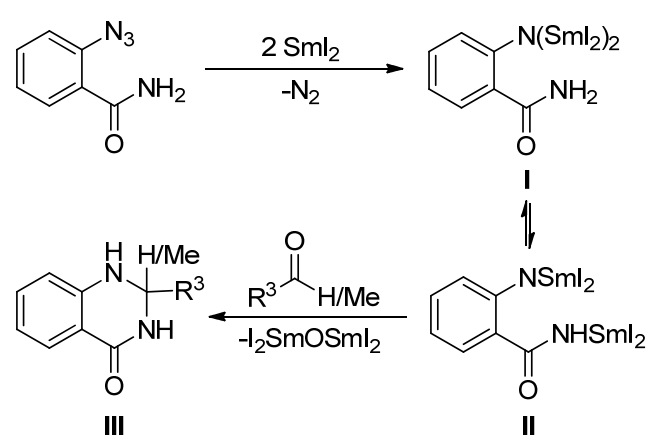

图式 $14 \mathrm{SmI}_{2}$ 作用下邻叠氮基苯甲酰胺与醛/酮反应生成 2,3二氢喹唑啉-4(1H)-酮的机理

Scheme 14 Proposed mechanism of reaction of $o$-azidobenzamide with aldehydes/ketones to 2,3-dihydroquinazolin-4-(1H)ones promoted by $\mathrm{SmI}_{2}$
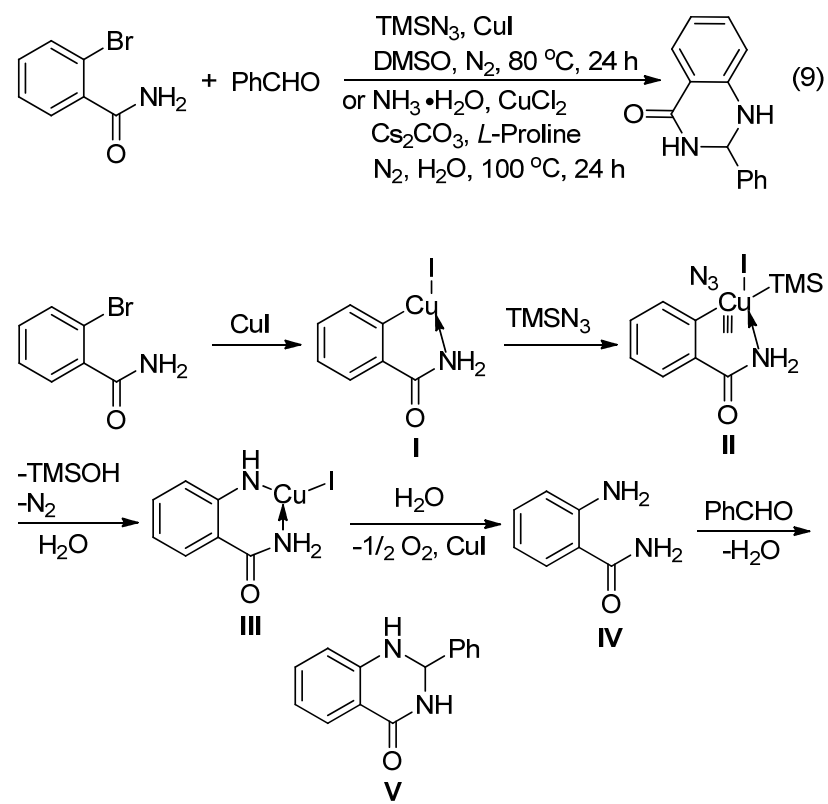

图式 15 邻溴苯甲酰胺与芳香醛反应生成 2,3-二氢喹唑啉4(1H)-酩的机理

Scheme 15 Proposed mechanism of reaction of $o$-bromobenzamide with aromatic aldehydes to 2,3-dihydroquina-zolin-4(1H)-ones

物 III, 再经还原消除反应转化为邻氨基苯甲酰胺 IV, 最后 IV 与芳香醛环合生成目标产物 $\mathbf{V}$.

\section{6 以邻溴苯甲腈为底物}

在 $\mathrm{CuCl}_{2}$ 催化下，邻溴苯甲腈与芳香醛、 $\mathrm{NH}_{3} \cdot \mathrm{H}_{2} \mathrm{O}$ 反应也生成 2-芳基取代的 2,3-二氢喹啉-4(1H)-酮类化合 物(Eq. 10 $)^{[63]}$. 该方法以 $L$-脯氨酸作配体, 以 $\mathrm{Cs}_{2} \mathrm{CO}_{3}$ 为 碱, 需要在氮气氛围中进行反应, 除 4-氯苯甲醛与邻溴 苯甲腈、 $\mathrm{NH}_{3} \cdot \mathrm{H}_{2} \mathrm{O}$ 反应能以 $96 \%$ 的收率得到目标产物外, 其余反应所得的目标产物的产率都普遍较低, 通常在 $0 \sim 77 \%$ 之间.

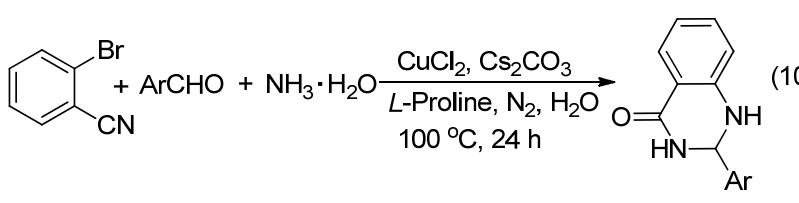

该反应的机理如 Scheme 16 所示. 在 $\mathrm{Cs}_{2} \mathrm{CO}_{3}$ 存在 下 $L$-脯氨酸与 $\mathrm{CuCl}_{2}$ 配位形成配合物 $\mathbf{I}$, 用以促进随后 与邻溴苯甲腈作用分别经 II、III 生成 2-氨基的乌尔曼 型反应，与此同时在 $\mathrm{CuCl}_{2} 、 \mathrm{Cs}_{2} \mathrm{CO}_{3}$ 和 $\mathrm{H}_{2} \mathrm{O}$ 存在下 $\mathrm{CN}$ 基被氧化为 $\mathrm{CONH}_{2}$ 基，即生成了中间体邻氨基苯甲酰 胺(IV), 随后 IV 再与芳香醛环合生成目标产物 $\mathbf{V}^{[63]}$.

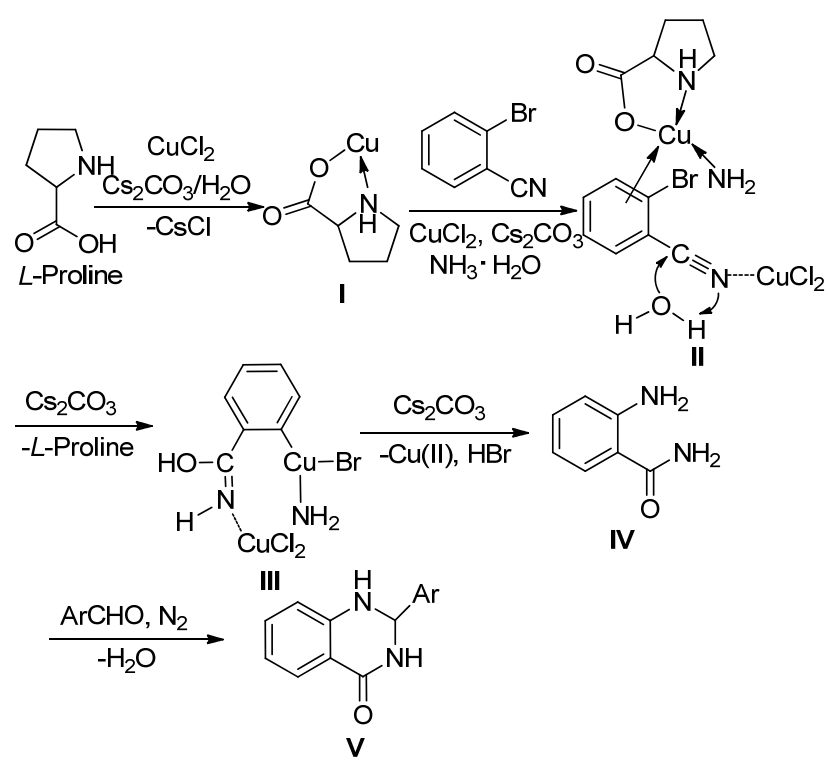

图式 16 邻溴苯甲腈与芳香醛和氨水反应生成 2,3-二氢喹唑 啉-4(1H)-酮的机理

Scheme 16 Proposed mechanism of reaction of $o$-bromobenzonitrile with aromatic aldehydes and aqueous ammonia to 2,3dihydroquinazolin-4-(1H)-ones

\section{7 以邻氨基苯甲酸为底物}

以正丙基膦酸酐( T3P)为缩合剂, $N$-取代邻氨基苯 甲酸可与环状亚胺在二异丙基乙胺(DIPEA)存在下反应 生成三环 2,3-二氢喹啉-4(1H)-酮类化合物 ${ }^{[64,65]}$. 该方法 所用试剂无毒, 对水和空气都不敏感, 反应条件温和, 操作方便, 只是反应需要进行 $20 \mathrm{~h}$, 用时相对较长. 当 环亚胺为 2,3,4,5-四氢吡啶、3,3-二甲基- $3 H$-吲哚时, 目 标产物的收率偏低，分别为 $40 \% 、 41 \%$; 其余环亚胺如 5,5-二苄基-2,3,4,5-四氢吡啶、4,4-二苄基-3,4-二氢- $2 H$ 吡咯、(3,4-二氢)异喹啉等与 $N$-甲基/苯基邻氨基苯甲酸 反应，均能很好进行，目标产物收率在 $87 \% \sim 97 \%$ 之间.

以 $N$-甲基邻氨基苯甲酸与 5,5-二芳基- $2,3,4,5$-四氢 吡啶反应为例, 二者首先反应生成 $N$-酰基亚胺离子中 间体，然后该中间体中的亚胺碳再接受氨基氮的亲核进 攻，环合生成目标产物(Scheme 17). 


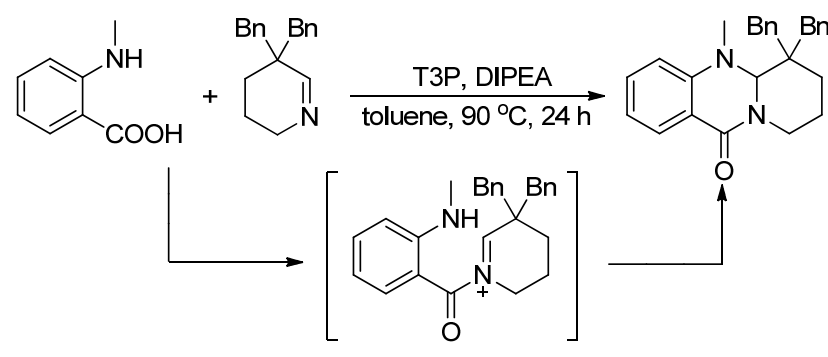

图式 17 邻氨基苯甲酸与环亚胺反应

Scheme 17 Reaction of $o$-aminobenzoic acids with cyclic imines

\section{8 以邻氨基苯甲腈为底物}

在催化剂作用下, 邻氨基苯甲腈可与醛/酮反应生 成 2,3-二氢喹啉-4(1H)-酮类化合物(Eq. 11). 所用催化 剂有 $\mathrm{K}_{3} \mathrm{PO}_{4}{ }^{[66]}$ 、胆碱 ${ }^{[67]}$ 、聚乙二醇键合的四乙基氢氧化

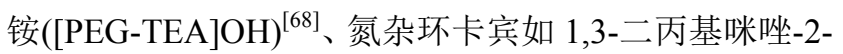
亚基(NHC-PPIm) ${ }^{[69,70]}$ 、大孔树脂 A26 OH (AA26OH) ${ }^{[71]}$ 、 金属改性的磺化硅胶 ${ }^{[72]}$ 等. 当醛参与反应时, 目标产物 收率良好，普遍在 $85 \% \sim 96 \%$ 之间; 酮参与反应时，产 率稍低，大多在 62\% 93\%之间.

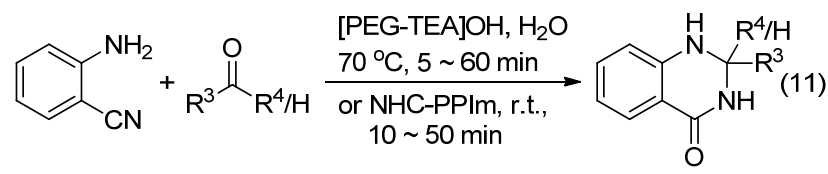

文献中报道的反应机理主要包括两条途径(Scheme 18): 其一, 在胆碱、 [PEG-TEA] OH 等催化剂活化作用
下，邻氨基苯甲腈中的氨基首先对醛/酮羰基进行亲核 进攻生成席夫碱中间体 IA, 紧接着 IA 中的氰基碳接受 来自催化剂中 $\mathrm{OH}^{-}$的亲核进攻从而由氰基经 IIA 转变 成相应的酰胺基(IIIA), 最后 IIIA 中的氨基在催化剂活 化作用下对其中的亚胺碳亲核进攻关环得到目标产物

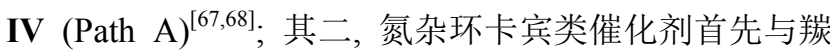
基化合物作用生成两性的布雷斯洛(Breslow)中间体 IB, 然后 IB 与邻氨基苯甲腈中的氰基作用生成另一种两性 离子 IIB, 随后 IIB 再关环脱去氮杂环卡宾的同时生成 相应的噁嗪中间体 IIIB，随后经分子内的 Dimroth 重排 反应生成目标产物 IV (Path B) ${ }^{[69,70]}$.

\section{9 以邻氨基 $N$-甲基- $N$-丙二烯基苯甲酰胺为底 物}

在 $\mathrm{Pd}(\mathrm{OAc})_{2}$ 催化下, 邻氨基 $N$-甲基- $N$-丙二烯基苯 甲酰胺可在室温下与芳基或乙烯基碘代烃反应生成相 应的在药物合成中具有重要应用的 2- $(\alpha$ - 芳基乙烯 基)-2,3-二氢喹啉-4(1H)-酮或 2-(2-1,3-丁二烯基)-2,3-二 氢喹啉-4(1H)-酮类化合物(Eq. 12 $)^{[73]}$. 该方法反应条件 温和, 绝大部分反应在 $0.25 \sim 2 \mathrm{~h}$ 内能够完成, 目标产物 收率在 39\% 94\%之间. 不足之处在于所用原料之一的 邻氨基 $N$-甲基- $N$-丙二烯基苯甲酰胺结构复杂、成本高 且来源不便; 此外, 包括另一原料碘代烃在内的两种起 始原料结构特殊, 多样性缺乏.

\section{0 以 $N$-烷基苯胺为底物}

最近，我们课题组开发了一种以结构简单、廉价易

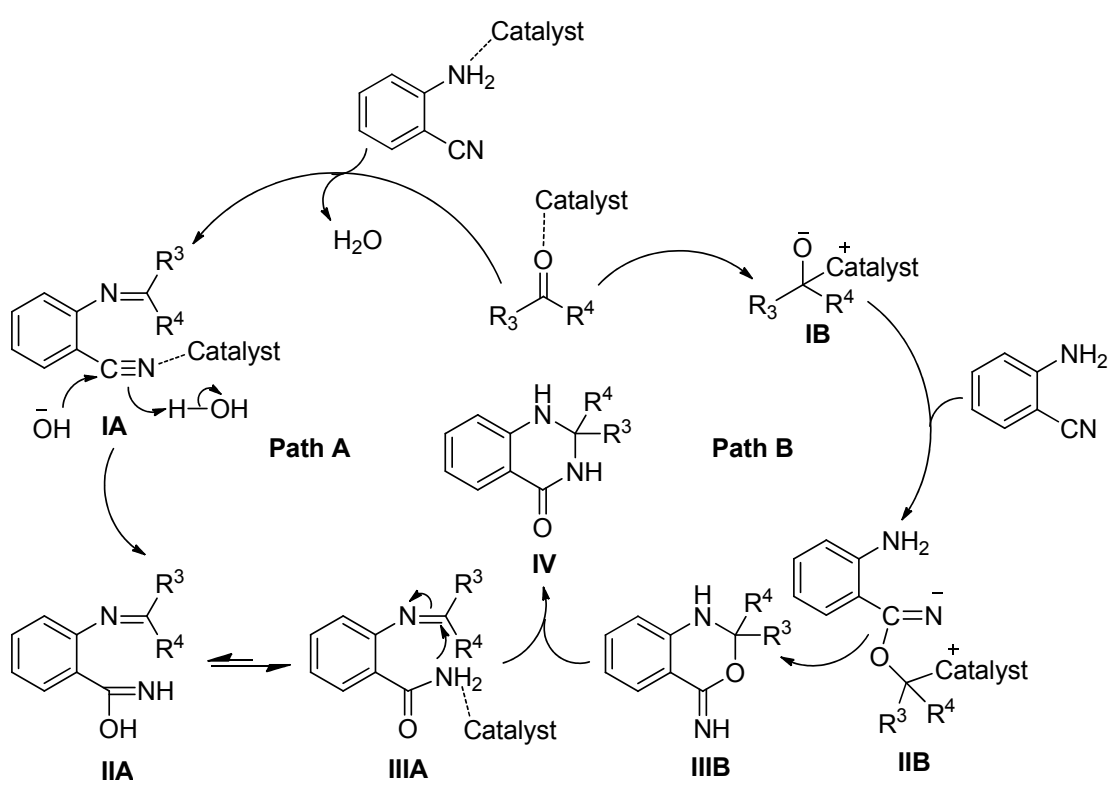

图式 18 邻氨基苯甲腈与醛/酮反应生成 2,3-二氢喹唑啉-4(1H)-酮的机理

Scheme 18 Proposed mechanism of reaction of $o$-aminobenzonitrile with aldehydes/ketones to 2,3-dihydroquinazolin-4-(1H)-ones 


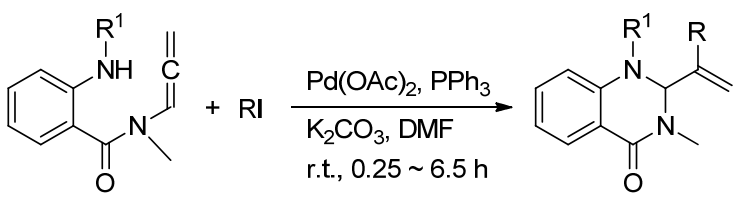

$\mathrm{R}^{1}=\mathrm{Ts} / \mathrm{COCF}_{3} / \mathrm{H} \quad \mathrm{R}=$ aryl/vinyl

得的 $N$-烷基苯胺为底物合成 2,3-二氢喹啉-4(1H)-酮类 化合物的新方法: 在 $\mathrm{Pd}(\mathrm{OAc})_{2}$ 催化下, $N$-烷基苯胺可与 $\mathrm{CO}$ 、胺、醛经氨基邻位 $\mathrm{C}-\mathrm{H}$ 键选择性活化, “一锅法” 多组分串联反应得到目标产物(Eq. 13 $)^{[74]}$.

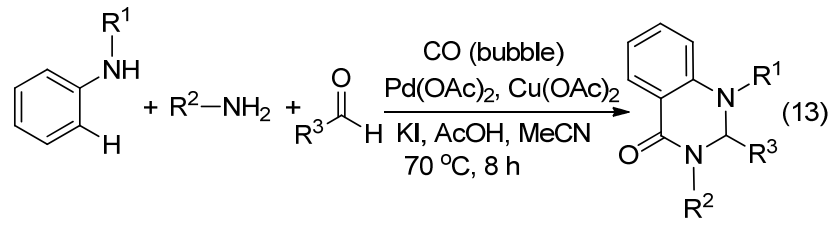

该反应的机理如 Scheme 19 所示. 在 $\mathrm{Pd}(\mathrm{OAc})_{2}$ 催化 下, $N$-烷基苯胺首先与 $\mathrm{CO}$ 作用, 分别经 I、II 转化为邻 氨基苯甲酸(III), 然后 III 再与 $\mathrm{Pd}(\mathrm{OAc})_{2} 、 \mathrm{CO}$ 作用, 经 $I V 、 V$ 转变为靛红酸䣶 $(V I)$. VI 在酸的活化作用下经 VII 胺解生成邻氨基苯甲酰胺(VIII), 最后 VIII 再与醛反应 关环生成目标产物 $\mathbf{I X}^{[74]}$.

\section{1 总结与展望}

鉴于 2,3-二氢喹唑啉-4(1H)-酮类化合物拥有广泛的 理及生物活性, 及其重要的应用价值, 该类化合物的合
成研究已取得长足的发展. 本文归纳总结的 10 大类方 法为该类化合物的主要合成方法，尽管各有优点，但不 同程度地存在着诸多问题. 例如: 所用起始原料结构复 杂，来源不便，原子经济性低，成本较高; 原料结构多 样性缺乏，导致产物结构通常比较单一，若想获得母体 骨架上带不同取代基的一系列喹唑啉酮衍生物，往往需 要进行后续反应，导致合成路线及反应时间较长，操作 繁琐; 反应条件苛刻，部分反应需在高温、无水、无氧 等条件下进行; 部分催化剂制备过程繁琐; 部分原料需 经制备后方可使用，目标产物产率不高. 此外，目前很 多研究工作主要致力于催化体系的研发上，而真正从源 头上探寻结构简单、廉价易得的原料来直接合成目标产 物的研究工作则鲜有报道. 我们课题组开发的钯催化 $N$ 烷基苯胺邻位 $\mathrm{C}-\mathrm{H}$ 键选择性活化 “一锅法” 多组分串 联反应合成 2,3-二氢喹唑啉-4(1H)-酮类化合物的方法, 较好地弥补了上述诸多合成方法存在的一些缺陷，具有 起始原料结构简单、多样性好且廉价易得、原子经济性 高、反应条件温和、操作简单、大部分目标产物的收率 从中等到良好等诸多优点，从而为该类化合物的合成开 辟出一条经济、高效的新途径. 美中不足的是，该方法 所用的催化体系尽管效果良好，但成本相对较高. 因此 继续探寻篮选简单、廉价、高效的催化体系，由结构简 单、廉价易得的原料经 “一锅者” 的多组分反应，简易、 经济、绿色合成 2,3-二氢喹唑啉-4(1H)-酮类化合物, 将 是今后化学工作者继续努力的方向.

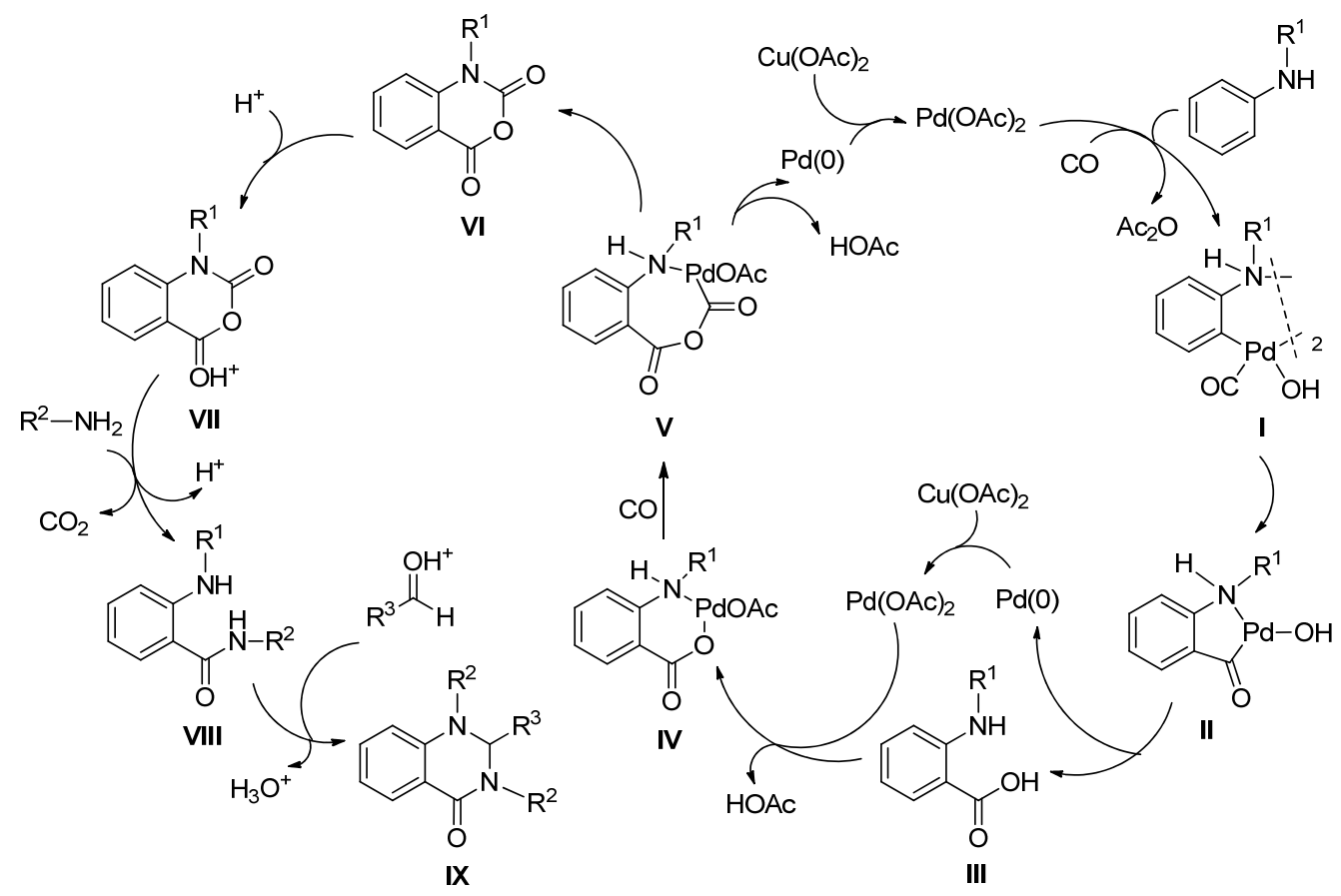

图式 $19 N$-烷基苯胺与 $\mathrm{CO}$ 、胺、醛反应生成 2,3-二氢喹唑啉-4(1H)-酮的机理

Scheme 19 Proposed mechanism of reaction of $N$-alkyl anilines with $\mathrm{CO}$, amines and aldehydes to 2,3-dihydroquinazolin-4-(1H)-ones 


\section{References}

[1] Kamble, A. A.; Kamble, R. R.; Chougala, L. S.; Kadadevarmath, J. S.; Maidur, S. R.; Patil, P. S.; Kumbar, M. N.; Marganakop, S. B. ChemistrySelect 2017, 2, 6882.

[2] Kamal, A.; Bharathi, E. V.; Reddy, J. S.; Ramaiah, M. J.; Dastagiri, D.; Reddy, M. K.; Viswanath, A.; Reddy, T. L.; Shaik, T. B.; Pushpavalli, S. N. C. V. L.; Bhadra, M. P. Eur. J. Med. Chem. 2011, 46, 691.

[3] Chinigo, G. M.; Paige, M.; Grindrod, S.; Hamel, E.; Dakshanamurthy, S.; Chruszcz, M.; Minor, W.; Brown, M. L. J. Med. Chem. 2008, 51,4620 .

[4] Roopan, S. M.; Khan, F. N.; Jin, J. S.; Kumar, R. S. Res. Chem. Intermed. 2011, 37, 919.

[5] Liao, C. H.; Pan, S. L.; Guh, J. H.; Chang, Y. L.; Pai, H. C.; Lin, C. H.; Teng, C. M. Carcinogenesis 2005, 26, 968.

[6] Hour, M. J.; Huang, L. J.; Kuo, S. C.; Xia, Y.; Bastow, K.; Nakanishi, Y.; Hamel, E.; Lee, K. H. J. Med. Chem. 2000, 43, 4479.

[7] Li, X. Q.; Wang, R. T.; Liu, Y.; Liu, Y.; Zheng, H.; Feng, Y. B.; Zhao, N.; Geng, H. B.; Zhang, W. Z.; Wen, A. D. BMC Pharmacol. Toxicol. 2017, 18, 73.

[8] Ibrahim, S. M.; Abo-Kul, M.; Soltan, M. K.; Barakat, W.; Helal, A. S. Med. Chem. 2014, 4, 351.

[9] Cheng, X.; Vellalath, S.; Goddard, R.; List, B. J. Am. Chem. Soc. 2008, 130, 15786

[10] Hemalatha, K.; Madhumitha, G.; Vasavi, C. S.; Munusami, P. J. Photochem. Photobiol. B 2015, 143, 139.

[11] Yu, H.; Jin, H. W.; Gong, W. Z.; Wang, Z. L.; Liang, H. P. Molecules 2013, 18, 1826.

[12] El-Sabbagh, O. I.; Ibrahim, S. M.; Baraka, M. M.; Kothayer, H. Arch. Pharm. Chem. Life Sci. 2010, 343, 274.

[13] Zhang, J.; Cheng, P.; Ma, Y. M.; Liu, J.; Miao, Z.; Ren, D. C.; Fan, C.; Liang, M.; Liu, L. Tetrahedron Lett. 2016, 57, 5271.

[14] Mohammadi, A. A.; Rohi, H.; Soorkic, A. A. J. Heterocycl. Chem. 2013, 50, 1129 .

[15] Xu, Z. H.; Zhang, Y. P.; Fu, H. C.; Zhong, H. M.; Hong, K.; Zhu, W. M. Bioorg. Med. Chem. Lett. 2011, 21, 4005.

[16] Ferrando, C.; Foy, J. M.; Pratt, C. N. F. W.; Purvis, J. R. J. Pharm. Pharmacol. 1981, 33, 219.

[17] Steinmuller, S. R.; Puschett, J. B. Kidney Int. 1972, 1, 169.

[18] Wang, Z. W.; Wang, M. X.; Yao, X.; Li, Y.; Tan, J.; Wang, L. Z.; Qiao, W. T.; Geng, Y. Q.;, Liu, Y. X.; Wang, Q. M. Eur. J. Med. Chem. 2012, 53, 275.

[19] Kothayer, H.; Ibrahim, S. M.; Soltan, M. K.; Rezq, S.; Mahmoud, S. S. Drug Dev. Res. 2019, 80, 343 ..

[20] Derbyshire, E. R.; Min, J.; Guiguemde, W. A.; Clark, J. A.; Connelly, M. C.; Magalhães, A. D.; Guy, R. K.; Clardy, J. Antimicrob. Agents Ch. 2014, 58, 1516.

[21] Hemalatha, K.; Madhumitha, G. J. Lumin. 2016, 178, 163.

[22] Singh, M.; Raghav, N. Bioorg. Chem. 2015, 59, 12.

[23] Sultana, N.; Sarfraz, M.; Tanoli, S. T.; Akram, M. S.; Sadiq, A.; Rashid, U.; Tariq, M. I. Bioorg. Chem. 2017, 72, 256.

[24] Sarfraz, M.; Sultana, N.; Rashid, U.; Akram, M. S.; Sadiq, A.; Tariq, M. I. Bioorg. Chem. 2017, 70, 237.

[25] Xing, J. H.; Yang, L. Y.; Yang, Y. F.; Zhao, L. L.; Wei, Q. Q.; Zhang, J.; Zhou, J. P.; Zhang, H. B. Eur. J. Med. Chem. 2017, 125, 411.

[26] Birch, H. L.; Buckley, G. M.; Davies, N.; Dyke, H. J.; Frost, E. J.; Gilbert, P. J.; Hannah, D. R.; Haughan, A. F.; Madigan, M. J.; Morgan, T.; Pitt, W. R.; Ratcliffe, A. J.; Ray, N. C.; Richard, M. D.; Sharpe, A.; Taylor, A. J.; Whitworth, J. M.; Williams, S. C. Bioorg. Med. Chem. Lett. 2005, 15, 5335.

[27] Katoh, T.; Takai, T.; Yukawa, T.; Tsukamoto, T.; Watanabe, E.; Mototani, H.; Arita, T.; Hayashi, H.; Nakagawa, H.; Klein, M. G.; Zou, H.; Sang, B. C.; Snell, G.; Nakada, Y. Bioorg. Med. Chem. 2016, 24, 2466.

[28] Zhang, H.; Liu, H.; Luo, X.; Wang, Y. X.; Liu, Y.; Jin, H. W.; Liu, Z. M.; Yang, W.; Yu, P. L.; Zhang, L. R.; Zhang, L. H. Eur. J. Med.
Chem. 2018, 152, 235

[29] Meinwald, Y. C.; Meinwald, J.; Eisner, T. Science 1966, 154, 390.

[30] Wang, X.; Yin, J.; Shi, L.; Zhang, G. P.; Song, B. A. Eur. J. Med. Chem. 2014, 77, 65.

[31] Giri, R.; Lam, J. K.; Yu, J. Q. J. Am. Chem. Soc. 2010, 132, 686.

[32] Diener, M. E.; Metrano, A. J.; Kusano, S.; Miller, S. J. J. Am. Chem. Soc. 2015, 137, 12369.

[33] Akyüz, G.; Menteşe, E.; Emirik, M.; Baltaş, N. Bioorg. Chem. 2018, 80,121 .

[34] Perreault, S.; Chandrasekhar, J.; Cui, Z. H.; Evarts, J.; Hao, J.; Kaplan, J. A.; Kashishian, A.; Keegan, K. S.; Kenney, T.; Koditek, D.; Lad, L.; Lepist, E. I.; McGrath, M. E.; Patel, L.; Phillips, B.; Therrien, J.; Treiberg, J.; Yahiaoui, A.; Phillips, G. J. Med. Chem. 2017, 60, 1555.

[35] Badolato, M.; Aiello, F.; Neamati, N. RSC Adv. 2018, 8, 20894.

[36] Cheng, X.; Vellalath, S.; Goddard, R.; List, Benjamin. J. Am. Chem. Soc. 2008, 130, 15786.

[37] Deng, T.; Wang, H. J.; Cai, C. J. Fluorine Chem. 2015, 169, 72.

[38] Tran, P. H.; Bui, T. P. T.; Lam, X. Q. B.; Nguyen, X. T. T. RSC Adv. 2018, $8,36392$.

[39] Sharma, M.; Pandey, S.; Chauhan, K.; Sharma, D.; Kumar, B.; Chauhan, P. M. S. J. Org. Chem. 2012, 77, 929.

[40] Sriramoju, V.; Kurva, S.; Chong, Y.; Madabhushi, S. New J. Chem. 2018, 42, 3188.

[41] Yamaguchi, K.; Kawaguchi, S.; Sonoda, M.; Tanimori, S.; Ogawa, A. Tetrahedron Lett. 2017, 58, 4043.

[42] Patil, N. T.; Lakshmi, P. G. V. V.; Singh, V. Eur. J. Org. Chem. 2010, 4719.

[43] Patil, N. T.; Kavthe, R. D.; Raut, V. S.; Shinde, V. S.; Sridhar, B. J. Org. Chem. 2010, 75, 1277.

[44] Sawatzky, E.; Wehle, S.; Kling, B.; Wendrich, J.; Bringmann, G.; Sotriffer, C. A.; Heilmann, J.; Decker, M. J. Med. Chem. 2016, 59, 2067.

[45] Noel, R.; Gupta, N.; Pons, V.; Goudet, A.; Garcia-Castillo, M. D.; Michau, A.; Martinez, J.; Buisson, D. A.; Johannes, L.; Gillet, D.; Barbier, J.; Cintrat, J. C. J. Med. Chem. 2013, 56, 3404.

[46] Razavi, N.; Akhlaghinia, B. New J. Chem. 2016, 40, 447.

[47] Zhang, J.; Ren, D. C.; Ma, Y. M.; Wang, W. T.; Wu, H. Tetrahedron 2014, 70, 5274.

[48] Carney, D. W.; Nelson, C. D. S.; Ferris, B. D.; Stevens, J. P.; Lipovsky, A.; Kazakov, T.; DiMaio, D.; Atwood, W. J.; Sello, J. K. Bioorg. Med. Chem. 2014, 22, 4836.

[49] Manivannan, E.; Chaturvedi, S. C. Bioorg. Med. Chem. 2012, 20, 7119.

[50] Jacob, E. D.; Mathew, L.; Thomas, B. J. Chem. Sci. 2007, 119, 47.

[51] Zhuang, Q. Y.; Fu, Y. C.; Tang, D.; Zha, Y. Y.; Rong, L. C.; Tu, S. J. Chin. J. Org. Chem. 2010, 30, 1405 (in Chinese). (庄启亚，付永春，唐丹，查云赟，荣良策，屠树江，有机化学， 2010, 30, 1405.)

[52] Darras, F. H.; Pockes, S.; Huang, G. Z.; Wehle, S.; Strasser, A.; Wittmann, H. J.; Nimczick, M.; Sotriffer, C. A.; Decker, M. ACS Chem. Neurosci. 2014, 5, 225.

[53] Huang, G. Z.; Roos, D.; Stadtmüller, P.; Decker, M. Tetrahedron Lett. 2014, 55, 3607.

[54] Rezaei, N.; Sheikhi, E.; Ranjbar, P. R. Synlett 2018, 29, 912.

[55] Azimi, S. B.; Azizian, J. Tetrahedron Lett. 2016, 57, 181.

[56] Bunce, R. A.; Nammalwar, B. J. Heterocycl. Chem. 2011, 48, 991.

[57] Shi, D. Q.; Rong, L. C.; Wang, J. X.; Zhuang, Q. Y.; Wang, X. D.; Hu, H. W. Tetrahedron Lett. 2003, 44, 3199.

[58] Cai, G. P.; Xu, X. L.; Li, Z. F.; Weber, W. P.; Lu, P. J. Heterocycl. Chem. 2002, 39, 1271

[59] Su, W. K.; Yang, B. B. Aust. J. Chem. 2002, 55, 695.

[60] Yoo, C. L.; Fettinger, J. C.; Kurth, M. J. J. Org. Chem. 2005, 70, 6941.

[61] Su, W. K.; Yang, B. B. J. Chem. Res., Synop. 2002, 604

[62] Upadhyaya, K.; Thakur, R. K.; Shukla, S. K.; Tripathi, R. P. J. Org. Chem. 2016, 81, 5046

[63] Liu, Z. B.; Zeng, L. Y.; Li, Chao.; Yang, F. B.; Qiu, F. S.; Liu, S. 
W.; Xi, B. M. Molecules 2018, 23, 2325.

[64] Unsworth, W. P.; Kitsiou, C.; Taylor, R. J. K. Org. Lett. 2013, 15, 258.

[65] Kitsiou, C.; Unsworth, W. P.; Coulthard, G.; Taylor, R. J. K. Tetrahedron 2014, 70, 7172.

[66] Wu, X. F.; Oschatz, S.; Block, A.; Spannenberg, A.; Langer, P. Org. Biomol. Chem. 2014, 12, 1865.

[67] Borase, P. N.; Thale, P. B.; Shankarling, G. S. RSC Adv. 2016, 6, 63078.

[68] Safaei, H. R.; Shekouhy, M.; Ghorbanzadeh, S. ChemistrySelect 2018, 3,4750
[69] Zhen, B.; Jiao, Q. Z.; Zhang, Y. P.; Wu, Q.; Li, H. S.; Shi, D. X.; Li, J. R. Catal. Commun. 2013, 32, 1.

[70] Chai, H. X.; Li, J. R.; Yang, L. P.; Liu, M. X.; Yang, D. L.; Zhang, Q.; Shi, D. X. Chin. J. Chem. 2014, 32, 865.

[71] Tamaddon, F.; Pouramini, F. Synlett 2014, 25, 1127.

[72] Zhang, L. J.; Yu, J. L.; Wang, W. L.; Li, H.; Xu, D. D.; Bi, Y. D.; Liu, F. D. Tetrahedron Lett. 2014, 55, 710.

[73] Kundu, P.; Mondal, M.; Chowdhury, C. J. Org. Chem. 2016, 81, 6596.

[74] Zhang, X. P.; Li, Z. W.; Ding, Q. Q.; Li, X. C.; Fan, X. S.; Zhang, G. S. Adv. Synth. Catal. 2019, 361, 976.

(Lu, Y.) 